\title{
芴基共价三嗪骨架聚合物的室温合成和取代基效应研究
}

\author{
于森 ${ }^{a}$ 徐雍捷 ${ }^{a}$ 蒋加兴*,b 任世杰*,a \\ $\left({ }^{a}\right.$ 四川大学高分子科学与工程学院 四川大学高分子材料工程国家重点实验室＼cjkstart成都 610065) \\ ( ${ }^{b}$ 陕西师范大学材料科学与工程学院 西安 710062)
}

\begin{abstract}
摘要 高效合成和功能性基团的引入是当前有机微孔聚合物材料研究的热点. 采用强质子酸催化的腈基三聚环化反 应，室温合成制备了一系列带有不同取代基的芴基共价三嗪骨架聚合物(FCTF1 FCTF3)，系统研究了取代基的变化对 所得材料光学性能、多孔性能及 $\mathrm{CO}_{2}$ 吸附能力的影响. 其中乙基取代的聚合物 FCTF2 具有最高的 BET 比表面积(621 $\left.\mathrm{m}^{2} / \mathrm{g}\right)$ 和 $\mathrm{CO}_{2}$ 吸附能力 $(1.8 \mathrm{mmol} / \mathrm{g}, 273 \mathrm{~K} / 1.1 \mathrm{bar})$. 该研究有助于加深对有机微孔聚合物结构与性能关系的理解, 对该类 材料的分子设计有借鉴意义.
\end{abstract}

关键词 有机微孔聚合物; 芴; 共价三嗪骨架聚合物; 多孔性; $\mathrm{CO}_{2}$ 吸附

\section{Room Temperature Synthesis and Substituent Effect Study of Fluorene-Based Covalent Triazine-Based Frameworks}

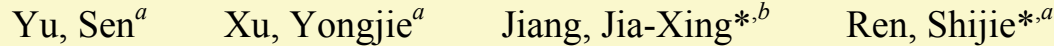 \\ $\left({ }^{a}\right.$ College of Polymer Science and Engineering, State Key Laboratory of Polymer Materials Engineering, Sichuan University, \\ Chengdu 610065) \\ ( ${ }^{b}$ School of Materials Science and Engineering, Shaanxi Normal University, Xi'an 710062)
}

\begin{abstract}
Efficient synthesis and the introduction of functional group are the focus of current research on microporous organic polymers (MOPs). In this report, a series of new covalent triazine-based framework polymers (CTFs) based on fluorene with different substituents (FCTF1 $\sim$ FCTF3) has been synthesized using trifluoromethanesulfonic acid (TFMS) catalyzed cyclotrimerization reactions at room temperature. The chemical structures of the polymers were confirmed by FTIR and elemental analysis. In the FTIR spectra, the nearly absence of peaks at around $2220 \mathrm{~cm}^{-1}$ along with the emergence of strong triazine absorption bands around 1500, 1360 and $800 \mathrm{~cm}^{-1}$ indicated qualitatively a high degree of polymerization. Thermogravimetric analysis (TGA) under nitrogen atmosphere revealed a high thermal stability with $5 \%$ weight loss at temperature up to 364 (FCTF1), 452 (FCTF2) and $238{ }^{\circ} \mathrm{C}$ (FCTF3). The solid UV-Vis spectra showed that the polymers could all absorb light from UV to visible light region. In the photoluminescence measurement, FCTF1 FCTF3 exhibited bright blue fluorescence with maximum emission wavelengths at $437 \mathrm{~nm}, 455 \mathrm{~nm}$ and $439 \mathrm{~nm}$ respectively. The specific surface areas of the polymers changed dramatically according to the substituent attached to the fluorine unit, with BET surface areas changing from nearly nil (FCTF3) to $621 \mathrm{~m}^{2} / \mathrm{g}$ (FCTF2) when the substituent changed from butyl to ethyl. Pore size distributions were calculated using nonlocal density functional theory (NL-DFT) and porous polymers FCTF1 and FCTF2 showed main pore sizes in the micropore region. $\mathrm{CO}_{2}$ adsorption capacities of the polymers were also measured and FCTF1 and FCTF2 showed high $\mathrm{CO}_{2}$ uptake of 1.7 and $1.8 \mathrm{mmol} / \mathrm{g}$ respectively at $273 \mathrm{~K} / 1.1$ bar. The isosteric heats of adsorption were calculated from the $\mathrm{CO}_{2}$ isotherms measured at 273 and $298 \mathrm{~K}$. FCTF1 and FCTF2 showed adsorption heats of 26.4 and $22.7 \mathrm{~kJ} / \mathrm{mol} \mathrm{respec}-$ tively at the zero coverage, indicative strong binding affinity of the polymers with $\mathrm{CO}_{2}$. To the best of our knowledge, this is the first report on the substituent effect of fluorene-based CTFs and this research can probably enhance the understanding of the structure-property relationship of porous organic polymer materials.

Keywords microporous organic polymers; fluorene; covalent triazine-based frameworks; porosity; $\mathrm{CO}_{2}$ adsorption
\end{abstract}

\section{1 引言}

有机微孔聚合物(MOPs)近年来因其在气体吸附、分 离及异相催化等领域的应用而广受关注 ${ }^{[1]}$. 与无机或有 机-无机杂化多孔材料如活性炭、沸石、金属氧化物和 金属-有机框架结构相比, MOPs 的优势在于同时具有合
成方法和性能的多样性以及良好的稳定性 ${ }^{[2]}$. 目前, 多 种类型的 MOPs 被广泛报道, 其中包括自具微孔聚合物 $(\mathrm{PIMs})^{[3]}$ 、有机共价骨架结构 $(\mathrm{COFs})^{[4]}$ 、共轭微孔聚合

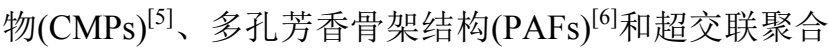
物(HCPs $)^{[7]}$ 等. 高效合成和功能性基团的引入是当前有

*E-mail: rensj@scu.edu.cn; jiaxing@snnu.edu.cn

Received February 2, 2015; published March 24, 2015.

Project supported by the National Natural Science Foundation of China (No. 21404074), the Specialized Research Fund for the Doctoral Program of Higher Education of China (No. 20130181120054) and the State Key Laboratory of Polymer Materials Engineering (Grant No. SKLPME2014-3-10).

项目受国家自然科学基金(No. 21404074)、教育部博士点基金(20130181120054)和高分子材料工程国家重点实验室自主课题(Sklpme2014-3-10)资助. 
机微孔聚合物材料研究的热点 ${ }^{[\mathrm{lb}]}$.

2008 年, Kuhn 等 ${ }^{[8]}$ 采用离子热手段在高温下 $(>$ $\left.400{ }^{\circ} \mathrm{C}\right)$ 以含有多个腈基的芳香族化合物的三聚环化反 应为基础合成了一系列基于均三嗪单元的高比表面积 有机多孔材料, 部分制备的三嗪基共价骨架聚合物 (CTFs)的 BET 比表面积大于 $3000 \mathrm{~m}^{2} / \mathrm{g}$. 此类聚合物的 合成单体便宜易得且在合成过程中避免了贵重过渡金 属催化剂的使用, 有较好的应用前景. 2009 年, 基于 2,6-二腈基吡啶的此类聚合物被用作甲烷转化为甲醇反 应的低温催化剂的载体 ${ }^{[9]} .2012$ 年, Sakaushi 等 ${ }^{[10]}$ 将无定 形的三嗪基多孔聚合物 ACTF-1 用作锂离子电池的正极 材料, 获得了具有高比能和高比功率的能量储存电极材 料. 最近, 任浩等 ${ }^{[11]}$ 使用类似反应定向合成了高效捕获 $\mathrm{CO}_{2}$ 的新型多孔芳香骨架材料.

然而, 上述合成方法需要长时间地使用较高的反应 温度 $\left(>400{ }^{\circ} \mathrm{C}\right)$, 很多功能化单体无法承受如此严苛的 反应条件且所得聚合物有碳化现象的出现，因此限制了 其实际使用. 2010 年, 表灵光课题组 ${ }^{[12]}$ 采用微波反应的 方法大大缩短了该反应的聚合时间. 最近, 王忠刚课题 组 ${ }^{[13]}$ 使用梯度加热的方法成功制备了基于杂环单体的 三嗪类多孔聚合物. 但是上述方法均使用 $\mathrm{ZnCl}_{2}$ 作为催 化剂, 后处理过程中 $\mathrm{ZnCl}_{2}$ 很难完全去除, 从而导致聚 合物的纯度降低. 2012 年, 我们发展了一种新的合成方 法制备此类聚合物, 该方法使用三氟甲磺酸(TFMS)作 为催化剂能够实现室温下此类聚合物的合成, 聚合反应 的单体容忍度及所得聚合物的纯度大大提高且其多孔 及光物理性能随使用单体的不同有规律性变化 ${ }^{[14]}$. 几 乎同时, Dai 课题组和刘洪来课题组合作也发展了 TFMS 催化的多腈基化合物三聚环化反应并制得多孔聚合物 薄膜用于 $\mathrm{CO}_{2}$ 的吸附与分离 ${ }^{[15]}$.

芴类有机及聚合物材料因其简单的合成方法, 良好 的加工性能和高的苂光量子效率被广泛应用于有机发 光二级管、有机太阳能电池等光电材料领域 ${ }^{[16]}$. 近年来, 含芴单元的多孔材料也相继有所报道. 基于多取代螺二 芴单元的超支化微孔聚合物具有较高的比表面积和较 窄的孔径分布, 可被用于氢气的吸附 ${ }^{[17]}$, 同时结合其稳 定的光学性能与多孔性能, 此类聚合物有望被应用于光 电器件的制备 ${ }^{[18]}$. 最近, 黄维课题组 ${ }^{[19]}$ 将含芴单元的配 体引入金属一有机骨架聚合物 (MOFs) 中, 并将此类 MOFs 用于含硝基爆炸物的荧光传感检测中.

然而, 将芴单元直接引入至有机微孔聚合物中的报 道很少, 其中一个原因是芴单元的官能化往往只在其 2、7 位两个位点上进行, 而共轭微孔聚合物的合成类似 于超支化聚合物的合成, 需要三官能度或更高的官能团 含量, 这一问题可以采用双官能团化的单体通过三聚反 应得以解决 ${ }^{[8,14]}$. 最近 Hug 等 ${ }^{[20]}$ 以 2,7-二腈基芴为单体 使用离子热反应在不同温度下合成出一系列 CTFs 类多 孔聚合物并详细研究了其对 $\mathrm{H}_{2}$ 和 $\mathrm{CO}_{2}$ 的吸附性能. 本
文中, 我们采用 TFMS 催化的腈基三聚环化反应室温下 制备了一系列带有不同烷基取代的芴类共价三嗪骨架 聚合物(FCTF1 FCTF3, 图 1), 系统研究了取代基的变 化对所得材料光学性能、多孔性能及 $\mathrm{CO}_{2}$ 吸附能力的影 响.
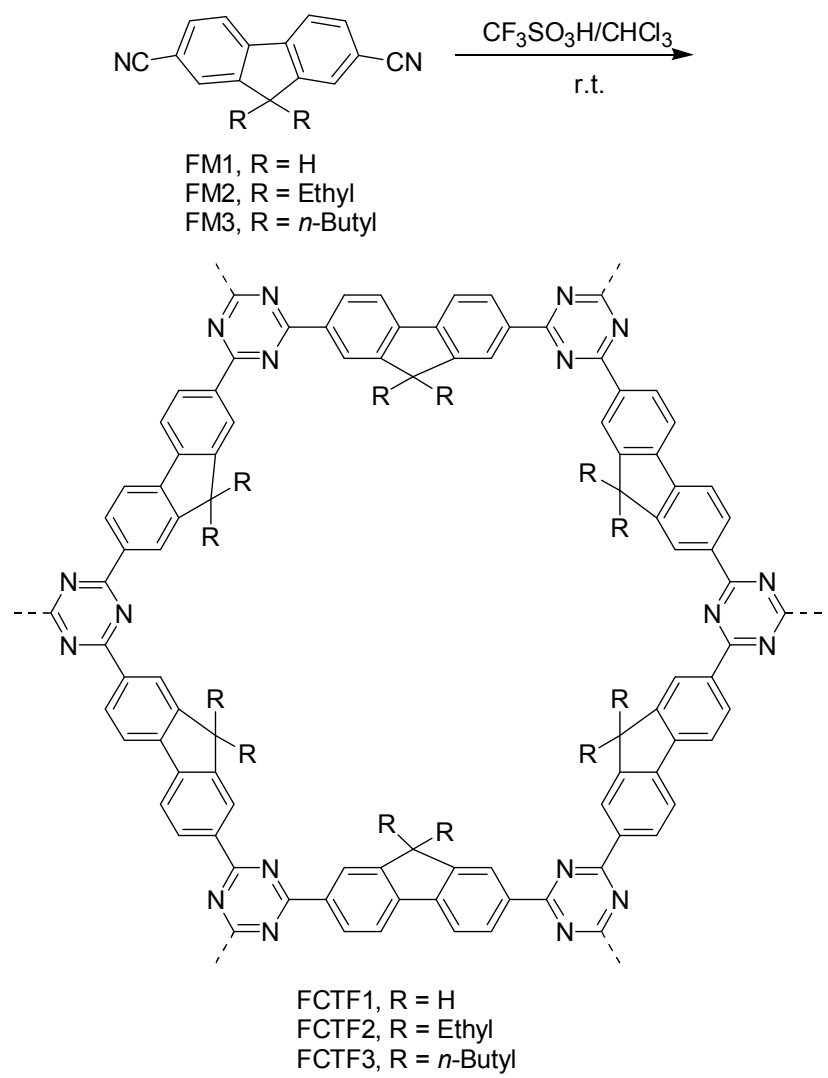

图 1 聚合物 FCTF1 TCTF3 的合成路线

Figure 1 Synthetic route of polymers FCTF1 FCTF3

\section{2 结果与讨论}

\section{1 聚合物 FCTF1 FCTF3 的合成与表征}

如图 1 所示, 分别使用 2,7-二腈基芴(FM1), 9,9-二 乙基-2,7-二腈基芴(FM2)以及 9,9-二正丁基-2,7-二腈基 芴(FM3)作为单体, TFMS 作为催化剂, 氯仿作为溶剂, 在室温下通过腈基三聚环化反应制得聚合物 FCTF1 FCTF3. 经多种溶剂洗涤、真空干燥后, 所得聚合物均 为粉末状固体, 且不溶于任何已知溶剂.

FCTF1 FCTF3 的红外光谱如图 $2 \mathrm{a}$ 所示, 单体分 子中腈基在 $2220 \mathrm{~cm}^{-1}$ 左右的特征峰基本消失，与此同 时, 三嗪单元在 1500,1360 和 $800 \mathrm{~cm}^{-1}$ 等处的特征峰明 显出现，定性地说明了聚合过程比较完全，聚合度较高. FCTF2 和 FCTF3 红外光谱中 $2800 \sim 2900$ 以及 $1250 、 750$ $\mathrm{cm}^{-1}$ 等处的特征峰说明聚合后烷基取代基的存在. 热 失重分析(图 2b)显示聚合物 FCTF1 FCTF2 具有较好 的热稳定性, 它们的 5\%热失重温度分别为 364 和 452 

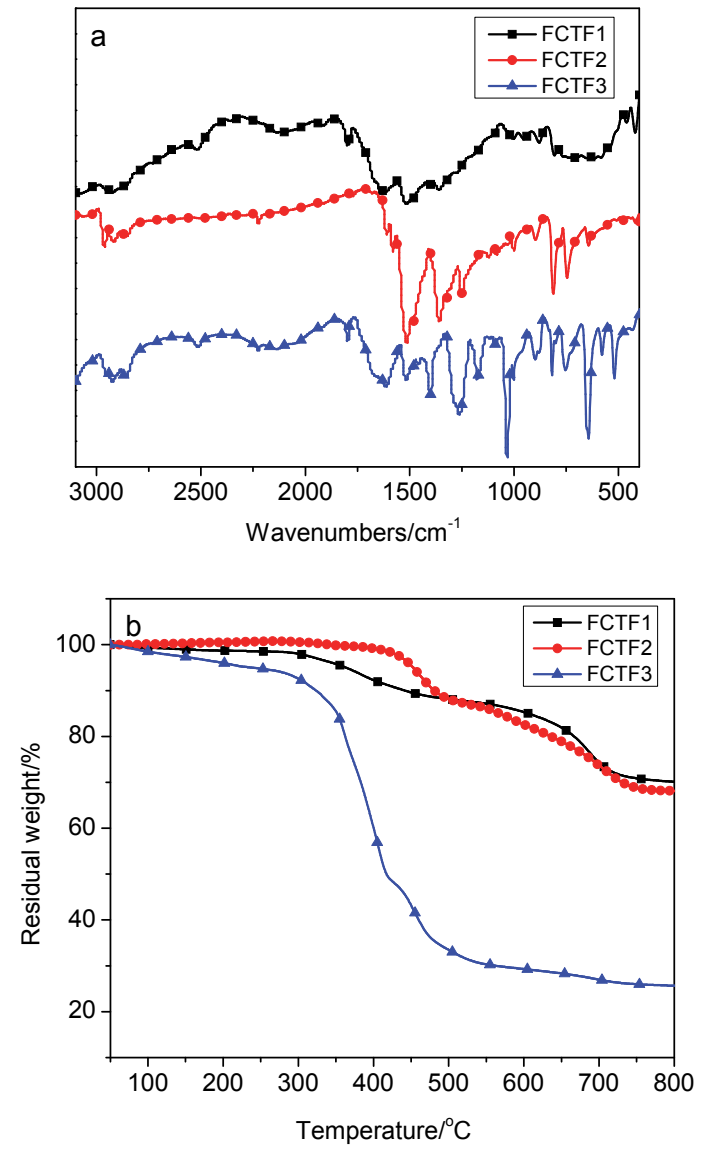

图 2 聚合物 FCTF1 FCTF3 的红外光谱图(a 图)和热失重曲线(b 图, 升温速度 $20 \mathrm{~K} / \mathrm{min}, \mathrm{N}_{2}$ 气氛)

Figure 2 FTIR spectra (a) and TGA curves (b, under $\mathrm{N}_{2}$, heating rate: $20 \mathrm{~K} / \mathrm{min}$ ) of polymers FTCF $1 \sim$ FCTF 3

${ }^{\circ} \mathrm{C}$. 相比于另外两个聚合物, FCTF3 的热稳定性较差 $\left(5 \%\right.$ 热失重温度仅为 $\left.238{ }^{\circ} \mathrm{C}\right)$, 这可能是因为所带取代 基较大，高温下容易分解造成.

\section{2 聚合物 FCTF1 FCTF3 的光学性能}

与离子热反应所得 $\mathrm{CTF}$ 类聚合物(一般为黑色固体) 不同，使用 TFMS 催化室温下制得的聚合物 FCTF1 FCTF3 均为淡黄色粉末状固体, 且在紫外灯下有明显 的蓝色荧光, 这些与芴类线形共轭聚合物相似. 固态紫 外-可见光谱(图 3a)显示 3 个聚合物在紫外到可见光区 域均有较宽的吸收范围且它们的最大吸收波长均在 300 $\mathrm{nm}$ 左右. 光致发光光谱如图 $3 b$ 所示, 聚合物 FCTF1 和 FCTF3 的最大发射波长相近, 均为 $435 \mathrm{~nm}$ 左右, 而带有 乙基的聚合物 FCTF2 的最大发射波长有较大红移, 在 $455 \mathrm{~nm}$ 左右, 这可能是因为 FCTF2 的聚合度较高, 这一 点在下面的多孔性能分析中也有所体现. 芴单元 9 位上 不带取代基的聚合物 FCTF1 的荧光光谱在 $550 \mathrm{~nm}$ 左右 出现了一个肩峰, 这与用于聚合物发光二极管领域的聚 芴类线形共轭聚合物发光纯度问题相似 ${ }^{[21]}$, 可能是由 于芴 9 位上未接取代基, 稳定性较差, 容易被氧化形成
芴酮结构所致.
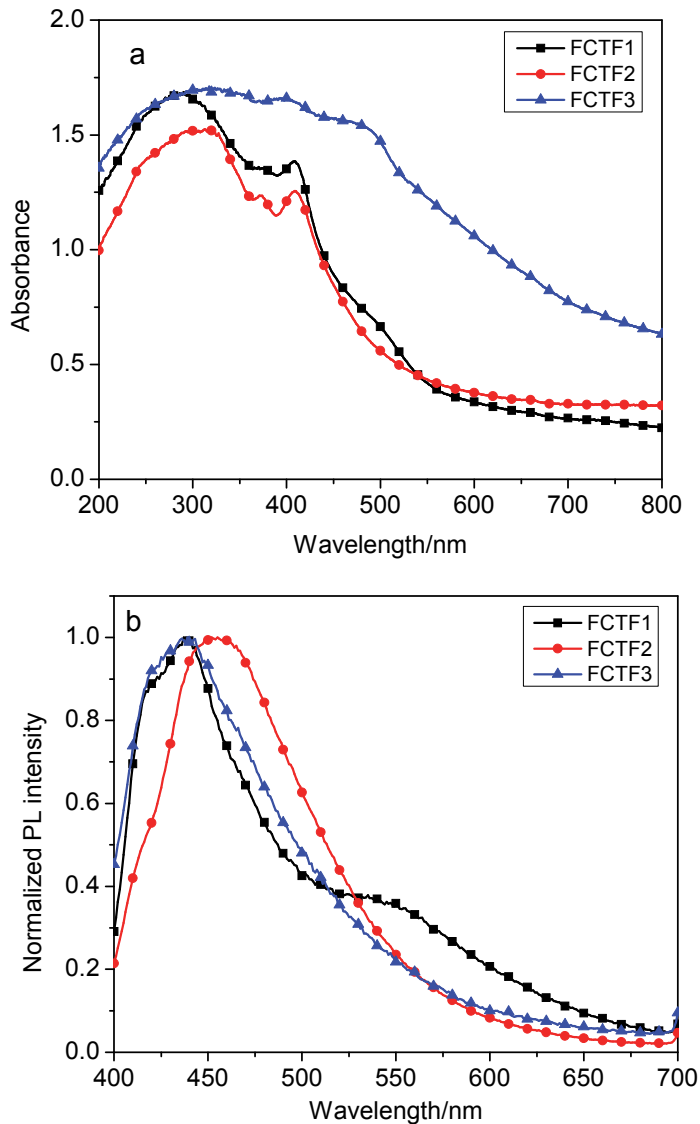

图 3 聚合物 FCTF1 FCTF3 的紫外-可见吸收光谱(a)和光致发光光 谱图(b, 激发波长: $360 \mathrm{~nm}$ )

Figure 3 UV-Vis adsorption spectra (a) and photoluminescence spectra (b, excitation wavelength: $360 \mathrm{~nm}$ ) of polymers FCTF1 $\sim$ FCTF3

\section{3 聚合物 FCTF1 FCTF3 的多孔性能}

聚合物 FCTF1 FCTF3 多孔性能通过氮气吸附 - 解 吸附实验测得(图 4a), 并应用 BET 模型算得聚合物的比 表面积. 芴单元 9 位上不带取代基的聚合物 FCTF1 和带 有乙基的聚合物 FCTF2 的 BET 比表面积分别为 268 和 $621 \mathrm{~m}^{2} / \mathrm{g}$, 而带有正丁基取代基的聚合物 FCTF3 比表面 积几乎为 0 . 荡单元 9 位不带取代基时, 聚合物中间体 稳定性差，且容易被氧化形成芴酮结构，聚合活性下降， 聚合度较低从而导致 FCTF1 的比表面积较低; 当单体 带有乙基取代基时，聚合物的稳定性提高，聚合较为完 全，比表面积也随之提高; 当取代基为体积更大的正丁 基时，柔性侧链容易造成聚合物孔拥堵，导致其比表面 积急剧降低. 聚合物 FCTF $1 \sim$ FCTF2 的等温吸附曲线均 为较典型的 Type I 吸附 ${ }^{[22]}$, 说明聚合物中主要是微孔结 构. 应用非局部密度泛函理论对聚合物的孔径分布进行 分析(图 4b), 聚合物 FCTF1 和 FCTF2 中主要存在着 $10 \sim 20 \AA$ 的微孔，聚合物 FCTF2 在 35 45 $\AA$ 区间内存 在少量的介孔. 

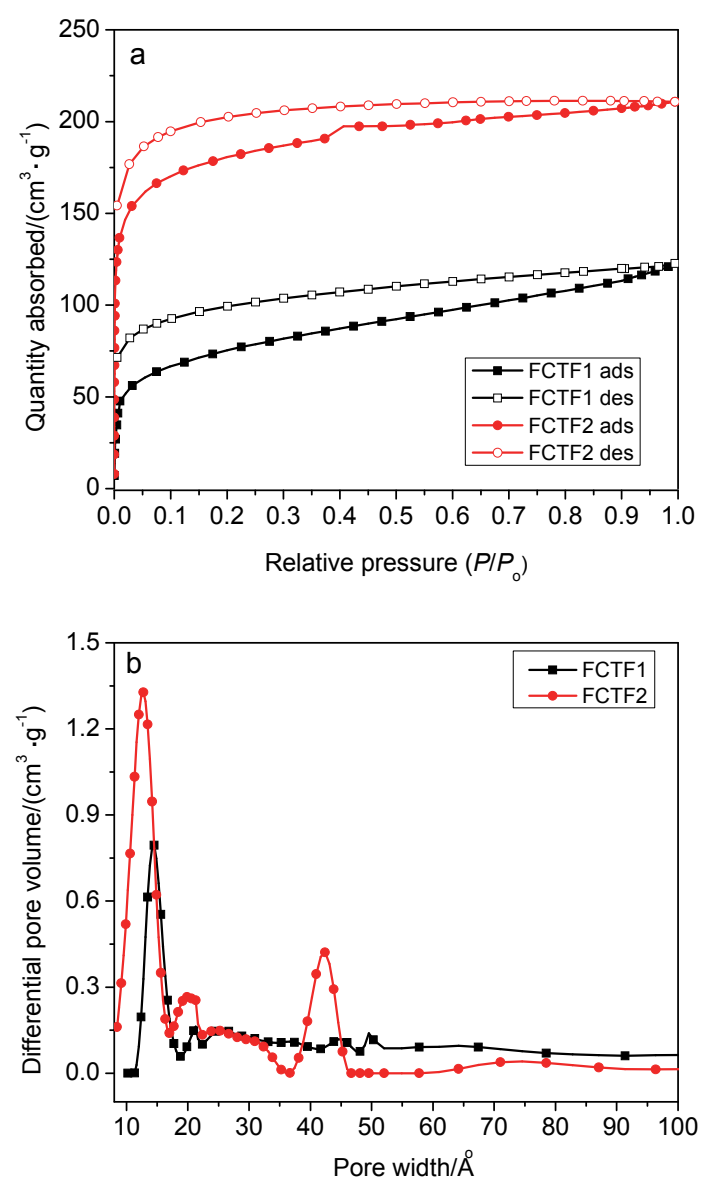

图 4 (a)聚合物 FCTF1 FCTF2 在 $77 \mathrm{~K}$ 的 $\mathrm{N}_{2}$ 吸附-脱附等温线(实心 标示为吸附, 空心标示为脱附); (b)聚合物 FCTF1 FCTF2 的孔径分 布曲线

Figure 4 (a) Nitrogen adsorption and desorption isotherms of polymers FCTF1 $\sim$ FCTF2 measured at $77 \mathrm{~K}$ (the adsorption and desorption branches are labeled with filled and empty symbols, respectively); (b) Pore size distribution curves of polymers FCTF1 $\sim$ FCTF2

\section{4 聚合物 FCTF1 FCTF2 的 $\mathrm{CO}_{2}$ 吸附性能}

$\mathrm{CO}_{2}$ 吸附分离是当前有机微孔聚合物的一个重要应 用方向, 为了得到具有高 $\mathrm{CO}_{2}$ 吸附能力的多孔材料, 关 于聚合物结构变化对其 $\mathrm{CO}_{2}$ 吸附性能影响的研究尤为 重要. 先前已经有较多研究关注聚合物骨架结构及元素 组成等对其 $\mathrm{CO}_{2}$ 吸附性能的影响, 而取代基效应的研究 相对较少 ${ }^{[23]}$. 芴结构单元良好的结构修饰性为取代基 效应的研究提供了很好的平台.

鉴于聚合物 FCTF1 FCTF2 具有较高的比表面积 和微孔结构, 我们对其在 273 和 $298 \mathrm{~K}$ 时的 $\mathrm{CO}_{2}$ 吸附性 能进行了测试. 在 $273 \mathrm{~K} / 1.1 \mathrm{bar}$ 条件下, 聚合物 FCTF1 和 FCTF2 的 $\mathrm{CO}_{2}$ 吸附量分别为 1.7 和 $1.8 \mathrm{mmol} / \mathrm{g}$ (图 5a); 在 $298 \mathrm{~K} / 1.1 \mathrm{bar}$ 条件下, 它们的 $\mathrm{CO}_{2}$ 吸附量分别为 1.1 和 $1.05 \mathrm{mmol} / \mathrm{g}$ (图 5b). 可见两个聚合物在不同温度下对 $\mathrm{CO}_{2}$ 的吸附能力相近, 而 FCTF1 的比表面积却比 FCTF2 低很多, 这说明相对 FCTF2 而言, FCTF1 与 $\mathrm{CO}_{2}$ 的相互
作用力更强. 为了进一步说明这一问题，我们使用两个 聚合物在 273 和 $298 \mathrm{~K}$ 的 $\mathrm{CO}_{2}$ 吸附数据应用 Clausius-Clapeyron方程计算其对 $\mathrm{CO}_{2}$ 的等量吸附热. 如 图 5c 所示, 在最小吸附量时, 聚合物 FCTF1 和 FCTF2 的 $\mathrm{CO}_{2}$ 吸附热分别为 26.4 和 $22.7 \mathrm{~kJ} / \mathrm{mol}$, 在整个吸附量 范围内, FCTF1 的 $\mathrm{CO}_{2}$ 吸附热均高于 FCTF2, 表明芴单
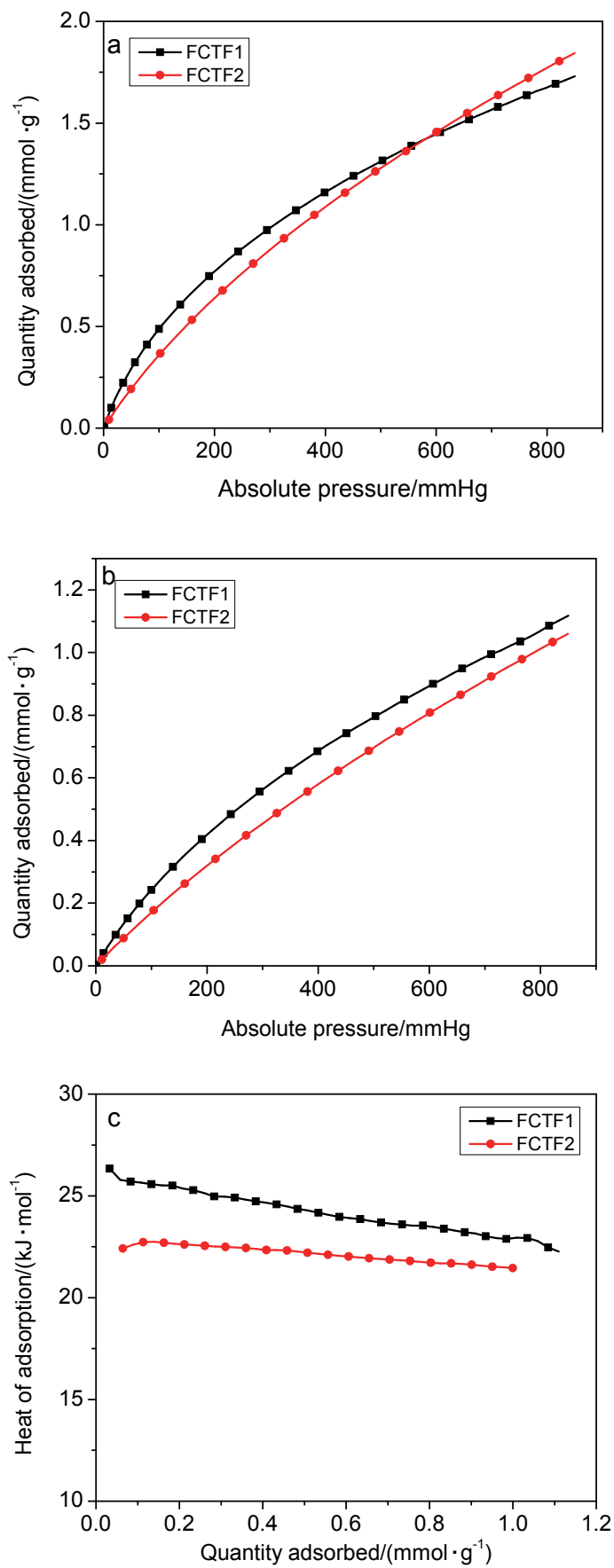

图 5 聚合物 FCTF1 FCTF2 在 $273 \mathrm{~K}$ (a)和 $298 \mathrm{~K}$ (b)的 $\mathrm{CO}_{2}$ 吸附等 温线及 $\mathrm{CO}_{2}$ 等量吸附热曲线(c)

Figure $5 \mathrm{CO}_{2}$ adsorption isotherms of FCTF1 FCTF2 at $273 \mathrm{~K}$ (a) and $298 \mathrm{~K}$ (b) and isosteric heats of adsorption for $\mathrm{CO}_{2}$ calculated from the adsorption isotherms collected at $273 \mathrm{~K}$ and $298 \mathrm{~K}$ (c) 
元 9 位上不带取代基时聚合物与 $\mathrm{CO}_{2}$ 的相互作用力更 强, 有利于聚合物对 $\mathrm{CO}_{2}$ 的吸附. 随着吸附量的增大, 两个聚合物对 $\mathrm{CO}_{2}$ 的吸附热降低均较为缓慢, 这与其微 孔结构相符.

\section{3 结论}

综上所述, 我们通过室温下 TFMS 催化的腈基三聚 环化反应合成了一系列取代基(分别为 $\mathrm{H}, \mathrm{Et}$ 和 $n$-Bu) 不 同的芴类三嗪基共价骨架聚合物 FCTF1、FCTF2 和 FCTF3, 并研究了芴 9 位取代基变化对所得聚合物光学 性能、多孔性能以及 $\mathrm{CO}_{2}$ 吸附性能的影响. 结果表明, 3 个聚合物在紫外到可见光区域均有较宽的吸收且均为 蓝光荧光材料. 随着取代基烷基链长度变长, FCTFs 的 比表面积呈先上升后下降的趋势, 其中带有乙基的 FCTF2 的比表面积为 $621 \mathrm{~m}^{2} / \mathrm{g}$, 其次为不带取代基的 FCTF1 为 $268 \mathrm{~m}^{2} / \mathrm{g}$, 而带有丁基的 FCTF3 则接近为 0 , 表明单体取代基的选择可有效调控聚合物的多孔性能. 聚合物 FCTF1 和 FCTF2 均表现出较好的 $\mathrm{CO}_{2}$ 吸附能力. $\mathrm{CO}_{2}$ 等量吸附热测试表明, 芴单元 9 位上不带取代基时 聚合物与 $\mathrm{CO}_{2}$ 之间的相互作用较强. 该研究是关于苆类 三嗪基共价骨架聚合物取代基效应的首次报道, 有助于 加深对有机微孔聚合物结构与性能关系的理解, 对该类 材料的分子设计有借鉴意义.

\section{4 实验部分}

聚合物 FCTF1 FCTF3 的合成: 在无水无氧的环境 下, 将单体 FM1 (FM2 或 FM3)溶解于无水氯仿中. 在 $0{ }^{\circ} \mathrm{C}$ 下, 将上述溶液缓慢滴加到三氟甲磺酸(TFMS)中, 自然升温至室温, 在室温下搅拌反应 $24 \mathrm{~h}$. 将反应液倒 入 $10 \%$ 的氨水溶液中搅拌 $1 \mathrm{~h}$, 过滤得到固体聚合物, 然后分别用去离子水、乙醇、甲醇、丙酮、氯仿充分洗 涤, 用氯仿索氏提取 $24 \mathrm{~h}$ 后真空干燥得到聚合物 FCTF1 (FCTF2 或 FCTF3). 产率 FCTF1, 99\%; FCTF2, 94\%; FCTF3, 99\%. FT-IR (KBr) v: FCTF1, 2930, 2223, 1780, 1675, 1610, 1578, 1480; FCTF2, 2980, 2223, 1520, 1460, 1350, 750; FCTF3, 2960, 2223, 1780, 1578, 1520, 1460, $1280,1050,650 \mathrm{~cm}^{-1}$.

\section{References}

[1] (a) McKeown, N. B.; Budd, P. M. Chem. Soc. Rev. 2006, 35, 675. (b) Dawson, R.; Cooper, A. I.; Adams, D. J. Prog. Polym. Sci. 2012, 37, 530. (c) Chang, Z.; Zhang, D.-S.; Chen, Q.; Bu, X.-H. Phys. Chem. Chem. Phys. 2013, 15, 5430. (d) Zhang, D.-S.; Chang, Z.; Lv, Y.-B.; Hu, T.-L.; Bu, X.-H. RSC Adv. 2012, 2, 408.

[2] Jiang, J.-X.; Cooper, A. Top. Curr. Chem. 2010, 293, 1.

[3] McKeown, N. B.; Gahnem, B.; Msayib, K. J.; Budd, P. M.; Tattershall, C. E.; Mahmood, K.; Tan, S.; Book, D.; Langmi, H. W.; Walton, A. Angew. Chem., Int. Ed. 2006, 45, 1804.

[4] El-Kaderi, H. M.; Hunt, J. R.; Mendoza-Cortés, J. L.; Côté, A. P.; Taylor, R. E.; O'Keeffe, M.; Yaghi, O. M. Science 2007, 316, 268.

[5] Jiang, J.-X.; Su, F.; Trewin, A.; Wood, C. D.; Campbell, N. L.; Niu, H.; Dickinson, C.; Ganin, A. Y.; Rosseinsky, M. J.; Khimyak, Y. Z.; Cooper, A. I. Angew. Chem., Int. Ed. 2007, 46, 8574.

[6] Ben, T.; Ren, H.; Ma, S.; Cao, D.; Lan, J.; Jing, X.; Wang, W.; Xu, J.; Deng, F.; Simmons, J. M.; Qiu, S.; Zhu, G. Angew. Chem., Int. Ed. 2009, 48, 9457 .

[7] Luo, Y.; Li, B.; Wang, W.; Wu, K.; Tan, B. Adv. Mater. 2012, 24, 5703.

[8] Kuhn, P.; Antonietti, M.; Thomas, A. Angew. Chem., Int. Ed. 2008, 47,3450 .

[9] Palkovits, R.; Antonietti, M.; Kuhn, P.; Thomas, A.; Schüth, F. Angew. Chem., Int. Ed. 2009, 48, 6909.

[10] Sakaushi, K.; Nickerl, G.; Wisser, F. M.; Nishio-Hamane, D.; Hosono, E.; Zhou, H.; Kaskel, S.; Eckert, J. Angew. Chem., Int. Ed. 2012, 51,7850

[11] Wang, W.; Yan, Z.-J.; Yuan, Y.; Sun, F.-X.; Zhao, M.; Ren, H.; Zhu, G.-S. Acta Chim. Sinica 2014, 72, 557. (王维，间卓君，元野，孙福 兴, 赵明, 任浩, 朱广山, 化学学报, 2014, 72, 557.)

[12] Zhang, W.; Li, C.; Yuan, Y.-P.; Qiu, L.-G.; Xie, A.-J.; Shen, Y.-H.; Zhu, J.-F. J. Mater. Chem. 2010, 20, 6413.

[13] Wu, S.-F.; Liu, Y.; Yu, G.-P.; Guan, J.-G.; Pan, C.-Y.; Du, Y.; Xiong, X.; Wang, Z.-G. Macromolecules 2014, 47, 2875.

[14] Ren, S.; Bojdys, M. J.; Dawson, R.; Laybourn, A.; Khimyak, Y.; Adams, D. J.; Cooper, A. I. Adv. Mater. 2012, 24, 2357.

[15] Zhu, X.; Tian, C.; Mahurin, S. M.; Chai, S.-H.; Wang, C.; Brown, S.; Veith, G. M.; Luo, H.; Liu, H.; Dai, S. J. Am. Chem. Soc. 2012 $134,10478$.

[16] Xie, L.-H.; Yin, C.-R.; Lai, W.-Y.; Fan, Q.-L.; Huang, W. Prog. Polym. Sci. 2012, 37, 1192.

[17] (a) Chen, Q.; Wang, J.-X.; Wang, Q.; Bian, N.; Li, Z.-H.; Yan, C.-G.; Han, B.-H. Macromolecules 2011, 44, 7987. (b) Yuan, S.; Kirklin, S.; Dorney, B.; Liu, D.-J.; Yu, L. Macromolecules 2009, 42, 1554.

[18] Weber, J.; Thomas, A. J. Am. Chem. Soc. 2008, 130, 6334.

[19] Zhou, X.; Li, H.; Xiao, H.; Li, L.; Zhao, Q.; Yang, T.; Zuo, J.; Huang, W. Dalton Trans. 2013, 42, 5718.

[20] Hug, S.; Mesch, M.-B.; Oh, H.; Popp, N.; Hirscher, M.; Senker, J.; Lotsch, B.-V. J. Mater. Chem. A 2014, 2, 5928.

[21] Ren, S.; Cheng, J.; Zeng, D.; Zhu, W.; Sun, J.; Du, J.; Xu, E.; Zhong, H.; Liu, Y.; Fang, Q. Synth. Met. 2009, 159, 29.

[22] Sing, K. S. W.; Everett, D. H.; Haul, R. A. W.; Moscou, L.; Pierotti, R. A.; Rouquerol, J.; Siemieniewska, T. Pure Appl. Chem. 1985, 57, 603.

[23] Qiao, S.; Du, Z.; Yang, R. J. Mater. Chem. A 2014, 2, 1877.

(Qin, X.) 